\title{
Atividades de extensão com cuidadores de pessoas com doença de Alzheimer: Desafios em tempos de COVID-19
}

Extension activities with caregivers of people with Alzheimer's disease: Challenges in times of COVID-19

Actividades de extensión con cuidadores de personas con enfermedad de Alzheimer: Desafíos en tiempos de COVID-19

\author{
Maíra de Oliveira Penna \\ https://orcid.org/0000-0002-3090-7055 \\ Universidade Franciscana, Brasil \\ E-mail: maira.penna@ufn.edu.br \\ Bruna Rodrigues Maziero \\ https://orcid.org/0000-0002-7680-0536 \\ Universidade Franciscana, Brasil \\ E-mail: brunarmaziero@gmail.com \\ Silomar Ilha \\ https://orcid.org/0000-0002-2132-9505 \\ Universidade Franciscana, Brasil \\ E-mail: silomar.ilha@ufn.edu.br \\ Tereza Cristina Blasi \\ https://orcid.org/0000-0002-5946-1640 \\ Universidade Franciscana, Brasil \\ E-mail: tcb29@hotmail.com \\ Jane Beatriz Limberger \\ https://orcid.org/0000-0002-8016-6742 \\ Universidade Franciscana, Brasil \\ E-mail: janebeatriz@ufn.edu.br
}

\begin{abstract}
Resumo
Este estudo objetivou relatar a experiência da organização das atividades extensionistas do grupo AMICA (Assistência Multiprofissional Integrada a Cuidadores de Pessoas com Doença de Alzheimer), em ambiente remoto durante o período de isolamento social devido a pandemia por COVID-19. Trata-se de um relato de experiência sobre as atividades extensionistas ocorridas no primeiro semestre de 2020. Foram realizadas diversas ações remotas de interação virtual, tais como envio de vídeos, músicas, panfletos informativos, e ligações telefônicas. Ainda, foram realizadas ações como o envio de cartas pelo correio e visitas domiciliares. Mesmo que por meio de ações remotas, foi possível dar voz ao cuidador, ouvir suas histórias, incentivar atividades físicas, trabalhar com educação em saúde e incentivar a sua socialização. Percebeu-se a importância das ações para a diminuição da distância entre os membros do grupo, redução da ansiedade e compreensão da importância do autocuidado. Adicionalmente, as atividades representaram uma relevante estratégia de educação em saúde durante a pandemia por COVID-19.
\end{abstract}

Palavras-chave: Relações comunidade-instituição; Idoso; Pandemia; Isolamento social; Pessoal de saúde.

\begin{abstract}
This study aimed to report the experience of organizing the extension activities of the AMICA group (Integrated Multiprofessional Assistance to Caregivers of People with Alzheimer's Disease), in a remote environment during the period of social isolation due to the COVID-19 pandemic. This is an experience report on the extension activities that took place in the first half of 2020. Several remote virtual interaction actions were carried out, such as sending videos, music, information pamphlets, and telephone calls. In addition, actions were carried out, such as mailing letters and home visits. Even though through remote actions, it was possible to give a voice to the caregiver, listen to their stories, encourage physical activities, work with health education and encourage their socialization. It was noticed the importance of actions to reduce the distance between the group members, reduce anxiety and understand the importance of self-care. Additionally, the activities represented a relevant health education strategy during the COVID-19 pandemic.
\end{abstract}

Keywords: Community-institutional relations; Elderly; Pandemic. Social isolation; Health personnel. 


\begin{abstract}
Resumen
Este estudio tuvo como objetivo reportar la experiencia de organizar las actividades de extensión del grupo AMICA (Asistencia Integrada Multiprofesional a Cuidadores de Personas con Enfermedad de Alzheimer), en un ambiente remoto durante el período de aislamiento social por la pandemia de COVID-19. Se trata de un informe de experiencia sobre las actividades de extensión que se llevaron a cabo en el primer semestre de 2020. Se realizaron varias acciones de interacción virtual remota, como envío de videos, música, folletos informativos y llamadas telefónicas. Además, se llevaron a cabo acciones, como envío de cartas y visitas domiciliarias. Aunque a través de acciones a distancia se logró dar voz al cuidador, escuchar sus historias, incentivar la actividad física, trabajar con la educación para la salud y fomentar su socialización. Se notó la importancia de las acciones para reducir la distancia entre los miembros del grupo, reducir la ansiedad y comprender la importancia del autocuidado. Además, las actividades representaron una estrategia de educación sanitaria relevante durante la pandemia de COVID-19.
\end{abstract}

Palabras clave: Relaciones comunidad-institucionales. Anciano; Pandemia; Aislamiento social; Personal sanitario.

\title{
1. Introdução
}

"O sofrimento só é intolerável quando ninguém cuida" (Cicely Saunders).

O envelhecimento populacional é um fenômeno mundial em crescimento significativo. No Brasil, ano de 2018, o Índice de Envelhecimento (IE) era de 63 idosos para cada 100 jovens e as projeções indicam que em 2055, para o mesmo número de jovens, o Brasil terá 202 idosos (IBGE, 2018). Embora o processo de envelhecimento não represente viver com doença, à medida que as pessoas envelhecem, torna-se susceptível ao surgimento de doenças crônicas-degenerativas, dentre as quais, destaca-se as que versam com demência (Santos et al., 2018).

A demência é caracterizada por um declínio progressivo da cognição e da funcionalidade, os quais conduzem às modificações comportamentais neuropsiquiátricas em relação a um nível prévio de desempenho (Bekhet, 2013). Tal condição, resulta na perda da independência para as atividades de vida diária (AVDs), que concluem: realizar a higiene corporal, vestirse e alimentar-se ou, ainda, ações mais complexas, como preparo das refeições, gerenciar o próprio dinheiro e o seu regime terapêutico, entre outros (Nunes et al., 2017)

Atualmente, cerca de 47 milhões de pessoas em todo o mundo apresentam algum tipo de demência, tornando-se uma das principais causas de dependência e incapacidade funcional. Em função do rápido envelhecimento da população, este número deve triplicar nos próximos 30 anos, gerando um elevado impacto socioeconômico, e exigindo dos países o desenvolvimento de políticas públicas específicas para esse grupo populacional, que possam reduzir o impacto ao indivíduo e suas famílias (Wolters et al., 2020).

Dentre as demências, destaca-se a doença de Alzheimer (DA) que, em termos patológicos, consiste no acúmulo de placas de proteína B-amiloide, formando emaranhados neurofibrilares contendo a proteína fosforilada Tau (p-Tau), o que conduz à angiopatia cerebral relacionada à queda do desempenho cognitivo e perda progressiva da memória recente (Morley e Nguyen, 2018).

O comprometimento da saúde da pessoa com DA é observado sobretudo por meio dos sintomas comportamentais e psicológicos, o que se reflete também na saúde do cuidador, seja ele familiar ou profissional. Neste contexto, as ações multiprofissionais interdisciplinares têm papel fundamental na manutenção do cuidado, tanto ao paciente quanto ao cuidador, evitando a fragmentação do cuidado (Bertazone et al., 2016).

Uma proposta interessante de cuidado interdisciplinar é a participação em grupos, que tem por função sensibilizar, acolher, vivenciar e compartilhar situações cotidianas de cuidado, contextualizando e resolvendo problemas que, em cada integrante, toma características particulares. Neste contexto, a participação em grupos melhora a qualidade de vida por meio do incentivo ao envelhecimento ativo e saudável (Fernandes et al., 2021). Nesse processo o sujeito é modificado pelo grupo, mas também provoca mudança (Silva et al., 2019). Baseados nesta premissa, o Grupo de Atenção Multiprofissional Integrada aos Cuidadores de Pessoas com Doença de Alzheimer (AMICA), construiu uma trajetória de cuidado nos últimos 15 anos, 
reunindo cuidadores familiares e profissionais, docentes e discentes da área da saúde a fim de promover a criação e efetivação de métodos interventivos que favoreçam a qualidade de vida (QV) de pessoas diagnosticadas com a doença e de seus familiares/cuidadores.

Em função da pandemia por COVID-19 no ano de 2020, as atividades cotidianas foram convertidas em encontros remotos, salvaguardando a segurança de todos os membros do grupo. Estas atividades são importantes para manter o vínculo entre todos os participantes e atender à situações pontuais que possam surgir durante a rotina do cuidado. Dessa forma, compreende-se a necessidade de socialização das atividades desenvolvidas, pois as mesmas podem incentivar e auxiliar a implementação de propostas semelhantes em diferentes contextos, fato que justifica a relevância desse estudo.

Justifica-se, ainda, por compreender que iniciativas relacionadas a saúde da pessoa idosa e das Doenças Crônicas Não Transmissíveis (DCNT), onde se insere a DA, são de grande importância no contexto de saúde, sendo destacadas como prioridades de pesquisa no Brasil (Brasil, 2018) Frente ao exposto, este artigo tem por objetivo relatar a experiência da organização das atividades extensionistas remotas durante o período de isolamento social devido a pandemia por COVID-19.

\section{Metodologia}

Trata-se de um relato de experiência da vivência de integrantes do grupo AMICA, junto aos familiares cuidadores de pessoas idosas com a DA, as quais foram reorganizadas por conta do distanciamento social vivenciado em decorrência da COVID-19. O relato de experiência configura-se como instrumento científico descritivo, que tem por finalidade apresentar a descrição de ações e ou procedimentos de forma contextualizada e reflexiva (Wang et al., 2020).

O Grupo AMICA caracteriza-se por um projeto de extensão, o qual desenvolve-se como um espaço de apoio a toda comunidade que busca o compartilhamento de conhecimentos e ajuda dentro do contexto da DA, uma vez que se propõe a desenvolver gerontotecnologias cuidativo-educacionais complexas para o cuidado integral. A população atendida consiste em famílias cuidadoras de idosos com a DA. Por gerontotecnologia complexa compreende-se: todo produto, processo, estratégias, serviço e/ou conhecimento, com a finalidade cuidativo-educacional da pessoa idosa e de seus familiares/cuidadores, fruto de uma construção/vivência coletiva complexa, que valorize as relações, interações e retroações dos envolvidos, por meio do conhecimento inter-multi-trans-meta-disciplinar (Ilha et al., 2017). O grupo realiza atividades com foco em ações transdisciplinares de educação em saúde, que compreende um saber ampliado e complexo em que diferentes áreas do saber formam juntas, um conhecimento comum, ou seja, trata-se de esquemas cognitivos que ultrapassam as disciplinas isoladas (Morin, 2010).

Além disso, o grupo é aberto para cuidadores formais e informais em busca de apoio e novos conhecimentos. O cuidador formal é considerando a pessoa maior de idade e que recebe remuneração para a atividade, com ou sem vínculo com uma instituição. Já, o informal é uma pessoa da família ou próxima do idoso, como um vizinho ou amigo que presta os cuidados, sem que haja qualquer tipo de contrato e pagamento (Diniz et al., 2018). Esse projeto de extensão está vinculado aos cursos da área da Saúde e a Pró-reitoria de Pesquisa e Relações Comunitárias da Universidade Franciscana, e se utiliza da infraestrutura da instituição para realização das atividades, oferecendo o respaldo que o grupo AMICA necessita para as suas atividades.

A metodologia das atividades ocorre por meio de encontros quinzenais com familiares/cuidadores e semanalmente com estudantes e docentes, no período vespertino, na sala do grupo AMICA e nos laboratórios de prática dos cursos envolvidos sempre que necessário. O número de participantes em cada reunião é variável, pois os mesmos são recebidos por livre demanda e os familiares/cuidadores novos são acolhidos com a finalidade de conhecer o binômio cuidador/paciente com DA e levantar suas necessidades.

Durante o primeiro semestre de 2020 , em função da necessidade de isolamento social imposto pela pandemia por 
COVID-19, foi necessário realizar um replanejamento das atividades em que foram priorizadas ações remotas por meio de plataformas digitais disponíveis, contando com a participação dos docentes, estudantes e familiares/cuidadores, no intuito de proporcionar cuidado e acolhimento por meio da abordagem de diferentes temáticas de interesse do grupo. Assim, foram elaboradas atividades por meio de vídeos e outros materiais digitais; realizadas ligações telefônicas e postagens em redes sociais (WhatsApp, Facebook, Instagram); visitas domiciliares (mantendo a distância física recomendada), envio de correspondências pelo correio, entre outros.

\section{Resultados e Discussão}

As atividades extensionistas no grupo AMICA procuram realçar a importância e a necessidade da dimensão humana no cuidado da dor e do sofrimento no âmbito da saúde de pessoas com DA. Muito se fala e se questiona sobre a desumanização do cuidado em saúde, especialmente das pessoas que necessitam de cuidados continuados e complexos. Há uma carência em termos de políticas públicas relacionadas com a humanização, bem como da qualificação do aparato formador de novos profissionais e criação de uma nova cultura que valorize os distintos saberes e trate com empatia o diferente (Pessini, 2009).

No contexto tecnológico imposto pela pandemia por COVID-19, se torna ainda mais relevante o resgate de uma visão antropológica holística, que cuide da dor e do sofrimento humano nas suas multidimensões, ou seja, que leve em conta os aspectos físicos, sociais, psicológicos, emocionais e espirituais. Vivenciar a dor e o sofrimento gerado pela convivência no contexto da doença crônica e degenerativa só é possível e suportável se o cuidado multidimensional for desenvolvido.

Para o paciente com DA e seu cuidador, é necessário modificar a perspectiva do adoecimento, de uma situação que vai levar à morte para uma situação em que se cuida e se aprimora a qualidade de vida (QV), baseado em descobertas científicas e no desenvolvimento de gerontotecnologias de cuidado. O conceito de gerontotecnologias abrange a interligação entre várias áreas da ciência com objetivo de utilizar uma assistência com tecnologias e cuidados às pessoas idosas e seus familiares cuidadores. Pode ser conceituada como o desenvolvimento tanto de produtos, como de ambientes e serviços para melhorar o cotidiano das pessoas idosas, com vistas a melhoria da QV (Ilha et al., 2018).

$\mathrm{O}$ reconhecimento da DA enquanto doença terminal permite antecipar as necessidades e problemas das pessoas acometidas. O grau de dependência em função da doença é variável, contudo, é possível inferir que a perda das capacidades cognitivas e funcionais resulte na necessidade de cuidados específicos. São comumente observados afasia, agnosia, apraxia, anomia, o comprometimento da marcha e do equilíbrio, além de sintomas psiquiátricos. Estes sintomas exigem conhecimentos específicos para promover a prestação de cuidados adequados, possibilitando a promoção da QV, a manutenção das funções, a maximização do conforto, o que contribui para um envelhecimento sustentável e digno (De Gois, 2019; Ximenes et al., 2014).

A proposta de "cuidar de quem cuida", norteadora das atividades do grupo AMICA, é ancorada nestes contextos e promove a reflexão sobre o papel do cuidador frente ao adoecimento da pessoa com DA. Trata-se de um ambiente rico de experiências, cujo compartilhamento auxilia na compreensão da doença e das diferentes formas de cuidar. Um estudo realizado com o objetivo de identificar gerontotecnologias desenvolvidas/empregadas pelos familiares/cuidadores como estratégias de cuidado complexo à pessoa idosa/família com doença de Alzheimer, identificou o grupo AMICA como sendo gerador de diferentes gerontotecnologias, na forma de produtos, processos, conhecimentos e estratégias, capazes de auxiliar o cuidador a enfrentar a rotina do cuidado da pessoa com DA (Ilha et al., 2018).

Por sua complexidade, o grupo AMICA caracteriza-se como uma Gerontotecnologia cuidativo-educacional complexa, geradora de novas gerontotecnologias de cuidado (Ilha et al., 2017). Frente a isso, os participantes do AMICA se reorganizaram a partir do isolamento social, de forma que as atividades do grupo, em 2020, foram priorizadas em ambiente virtual. As temáticas trabalhadas no referido ano envolveram o cuidado na prevenção da COVID-19, acolhimento, espiritualidade, autocuidado, aspectos biopsicossociais que possam estar sendo negligenciados no período de pandemia. 
Para tanto, com objetivo de promover a melhora dos aspectos psicológicos do cuidado e do acolhimento, os professores, bolsistas e convidados foram incumbidos de gravar vídeos de até cinco minutos sobre diferentes temáticas e compartilhar via grupo de WhatsApp. Também foram utilizados clips musicais disponíveis na web, cujas temáticas estivessem voltadas ao bem estar dos participantes do grupo. O uso de vídeos enquanto ferramenta de contato virtual possui a vantagem de atingir um grande alcance, elevado interesse de compartilhamento e excelente potencial pedagógico para educação em saúde. A música é um elemento criativo e humanizado, que possui um imenso potencial como redutor de estresse, ansiedade e dor; aumento da QV de pacientes e familiares e melhora das relações interpessoais, valorizando o cuidado do corpo-mente-alma da pessoa humana (Nunes et al., 2020).

Outra estratégia utilizada pelos participantes do AMICA foi a utilização de cartilhas e folhetos digitais a fim de considerar aspectos mais técnicos do cuidado. Para tanto, além de materiais informativos institucionais produzidos por órgãos oficiais, como o Ministério da Saúde e associações profissionais, foram construídos diferentes dispositivos de informação que contemplassem as necessidades apontadas pelos próprios familiares/cuidadores participantes do grupo, como por exemplo, a higienização de alimentos, o uso de medicamentos e suplementos alimentares, a prática de atividades físicas, etc. Tais materiais, tratam-se de tecnologias leve-duras, que compreendem conhecimentos técnico-científicos específicos apresentados na forma de recursos pedagógicos e que servem de subsídio para o manejo grupal (Silva, 2013).

Em outro momento, a estratégias adotada pelos membros do AMICA foi a escrita de cartas a próprio punho e o envio aos familiares/cuidadores pelo correio. Essa estratégia surgiu a partir da compreensão de que em tempos de correio eletrônico, é possível identificar grandes mudanças no processo de transmissão e recepção de mensagens, em relação ao que era realizado por meio das cartas tradicionais. Contudo, entende-se que as cartas tradicionais continham muitas vezes relatos detalhados e minuciosos do dia a dia das pessoas. Muitas coleções de cartas foram até reunidas em livros, capazes de contar histórias que atravessavam gerações (Santos, 2007). Assim, identificou-se que o fato de receber uma carta pelo correio levou muitos cuidadores a vivenciar um retorno ao passado, e a emoção foi descrita com lágrimas de satisfação e saudade, personalizando os afetos.

Outra estratégia empregada foi o contato telefônico, pois compreendeu-se que o mesmo poderia apresentar vantagens como a personalização do cuidado. Essa estratégia possibilitou responder aos anseios pessoais de cada cuidador, principalmente sobre questões imediatas e inadiáveis. O telefonema do familiar se configura como ajuda em meio a uma crise, que pode estar relacionada ao enfrentamento do diagnóstico e aos cuidados exigidos pela pessoa com DA. O atendimento telefônico acarreta um tipo de demanda que exige escutar sensivelmente o que o familiar tem a dizer; responder a seus questionamentos, informá-lo adequadamente sobre as atividades do grupo ou sobre a própria doença e a rotina do cuidado (Xavier e Alvarez, 2006).

Além disso, outra estratégia foi a utilização do grupo de Whatsapp, onde os cuidadores foram estimulados a relatar suas atividades diárias por meio da postagem de fotos, mostrando um pouco de sua rotina familiar e de cuidado. Neste cenário, o compartilhamento atua como potencializador de vínculos (Carvalho et al., 2006). Trata-se de uma forma de reforçar a convivialidade e a percepção de que todos estão passando por dificuldades semelhantes, mas que com o apoio mútuo são capazes de implementar mudanças no dia a dia.

Foram realizadas, ainda, interação por meio de visitas domiciliares, nas quais foi entregue uma máscara de tecido confeccionada pelos componentes do grupo, a cada um dos participantes. A visita domiciliar pode ser conceituada como um instrumento para inserção e conhecimento do contexto de vida de determinada população, bem como para o estabelecimento de vínculos entre profissionais e usuário (Kebian e Acioli, 2014), nesse caso, os familiares/cuidadores de pessoas com a DA. $\mathrm{Na}$ visita domiciliar podem ser desenvolvidas ações de orientação, educação, levantamento de possíveis soluções de saúde, fornecimento de subsídios educativos, para que as pessoas atendidas tenham condições de se tornar independentes (Kebian e 
Acioli, 2014).

Esta ação obedeceu aos critérios de distanciamento social, priorizando o contato breve, porém, pautado no afeto destas relações interpessoais. Para tanto, o grupo de docentes e discentes participantes do AMICA, separam-se em duplas ou trios para as visitas, de forma que não houvesse aglomerações. A entrega da máscara foi realizada, na maioria das vezes, na porta da casa, momento em que os docentes e discentes perguntam como estavam os familiares/cuidadores da pessoa idosa com a DA. Nessa ação, foi possível o compartilhamento de olhares carinhosos e a certeza de que nenhum dos participantes estava sozinho, pois o AMICA estava com todos e disponível a auxiliá-los dentro das possibilidades e das limitações impostas pela pandemia da COVID-19.

Destaca-se, por fim, que as atividades desenvolvidas procuraram atender às necessidades e o momento de vida de cada um dos cuidadores, favorecendo a atenção transdisciplinar, humanizada e afetiva, proporcionando experiências de educação em saúde em diferentes níveis de complexidade.

\section{Conclusão}

As vivências de um grupo de extensão em um momento de isolamento social imposto pela pandemia por COVID-19 aqui relatadas evidenciaram um sentimento de aproximação emocionante, mostrando que todos estavam perto, apesar de longe. Neste momento em que se destaca a preocupação com a saúde dos idosos, população de maior risco de morbimortalidade pela doença, provoca a necessidade de ampliação das ações de proteção, respeito, dignidade e rede de apoio. As reflexões desenvolvidas aqui buscam alertar pontos fundamentais para discussões referentes a integridade da pessoa idosa e dos familiares/cuidadores, a partir das suas subjetividades, integrando-a na medida do possível à sociedade. Muito além dos esforços para levar conteúdos informativos e de fontes confiáveis, o feedback dos participantes serve de estímulo para a continuação de trabalhos deste tipo neste contexto incerto.

\section{Referências}

Beckhet, A. K. (2013). Effectos of positive cognitions and resourcefulness on caregiver burden among caregiver os persons with dementia. Int J ment Health Nurs., 22(4), 340-346, 2013.

Bertazone, T. M. A., Ducatti, M., de Camargo, H. P. M., Batista, J. M. F., Kusumota, L., \& Marques, S. (2016). Ações multidisciplinares/interdisciplinares no cuidado ao idoso com Doença de Alzheimer. Revista da Rede de Enfermagem do Nordeste, 17(1), 144-153.

Carvalho, A., Bastos, A. C. S., Rabinovich, E. P., \& Sampaio, S. M. (2006). Vínculos e redes sociais em contextos familiares e institucionais: uma reflexão conceitual. Psicologia em estudo, 11(3), 589-598.

Diniz, M. A. A., Melo, B. R. D. S., Neri, K. H., Casemiro, F. G., Figueiredo, L. C., Gaioli, C. C. L. D. O., \& Gratão, A. C. M. (2018). Estudo comparativo entre cuidadores formais e informais de idosos. Ciência \& Saúde Coletiva, 23, 3789-3798.

Fernandes, D. de S., Moraes, J. L. A., Bandeira, G. de L. S., Azevedo Júnior, W. S., Procópio, T. B., Freitas, E. F., \& Gonçalves, L. H. T. (2021). A extensão universitária e sua relação com a qualidade de vida de idosos vulneráveis amazônidas. Research, Society and Development, 10(3), e14810313240

Góis, B. P. (2019). Promoção do bem-estar subjetivo em pessoas idosas com demência: Terapia da reminiscência. Dissertação de Mestrado. Faculdade de Medicina da Universidade do Porto. 135 p.

Ilha, S., Santos, S. S. C., Backes, D. S., Barros, E. J. L., Pelzer, M. T., \& Oliveira, A. M. N. D. (2017). Educational and care-related (geronto) technology in Alzheimer's disease and in supporting the elderly/family: perspective of teachers and students. Escola Anna Nery, 21(2), 1-8.

Ilha, S., Santos, S.S.C., Backes, D.S. Amica: (geronto)tecnologia cuidativo-educacional complexa no contexto da doença de Alzheimer. 1 ed. Curitiba: CRV, 2017.

Ilha, S., Santos, S. S. C., Backes, D. S., Barros, E. J. L., Pelzer, M. T., \& Gautério-Abreu, D. P. (2018). Gerontotecnologias utilizadas pelos familiares/cuidadores de idosos com alzheimer: contribuição ao cuidado complexo. Texto \& Contexto-Enfermagem, 27(4).

Instituto Brasileiro de Geografia e Estatística (IBGE). Tabela 2010-2060 - Projeção da População (revisão 2018), Rio de Janeiro: IBGE, 2018. Disponível em: https://agenciadenoticias.ibge.gov.br/agencia-sala-de-imprensa/2013-agencia-de-noticias/releases/21837-projecao-da-populacao-2018-numero-de-habitantesdo-pais-deve-parar-de-crescer-em-2047

Kebian, L. V. A., \& Acioli, S. (2014). A visita domiciliar de enfermeiros e agentes comunitários de saúde da Estratégia Saúde da Família. Revista Eletrônica de Enfermagem, 16(1), 161-9. 
Research, Society and Development, v. 10, n. 5, e31510514967, 2021

(CC BY 4.0) | ISSN 2525-3409 | DOI: http://dx.doi.org/10.33448/rsd-v10i5.14967

Lopes, R. F., Soares, M. S., de Sá, L. F., \& Câmara, V. D. (2009). Toque: ferramenta terapêutica no tratamento geriátrico e gerontológico. Revista Brasileira de Ciências do Envelhecimento Humano, 6(3).

Morin, E. A cabeça bem-feita: repensar a reforma, reformar o pensamento. 17. Ed. Rio de Janeiro: Bertrand Brasil, 2010. 128p.

Morley, J. E., Farr, S. A., \& Nguyen, A. D. (2018). Alzheimer disease. Clinics in geriatric medicine, 34(4), 591-601.

Nunes, D. L. D. S., Fontes, W. D. S., \& De Lima, M. A. (2017). Cuidado de enfermagem ao paciente vítima de acidente vascular encefálico. Revista Brasileira de Ciências da Saúde, 21(1), 87-96.

Nunes, Emanuelle Caires Dias Araújo et al. A música como instrumento de cuidado transpessoal-percepções de indivíduos hospitalizados assistidos na extensão universitária. Escola Anna Nery, v. 24, n. 2, 2020.

Osorio, L. C. Grupoterapias: abordagens atuais (2008). Porto Alegre: Artmed.

Pessini, L. (2009). Humanização da dor e sofrimento humanos no contexto hospitalar. Revista Bioética, 10(2).

Santos, F. H., Watchman, K., \& Janicki, M. P. (2018). Highlights from the International Summit on Intellectual Disability and Dementia Implications for Brazil. Dementia \& neuropsychologia, 12(4), 329-336.

Santos, R. M. Que clique é esse? Um estudo comparativo entre o correio eletrônico e o correio tradicional. (2007). Intercom - Sociedade Brasileira de Estudos Interdisciplinares da Comunicação V Congresso Nacional de História da Mídia.

Silva, D. M. G. V. D., Meirelles, B. H. S., \& Zanetti, M. L. (2013). Curso de Especialização em Linhas do Cuidado em Enfermag em: tecnologias do cuidado em saúde. In Curso de Especialização em Linhas do Cuidado em Enfermagem: tecnologias do cuidado em saúde (pp. 126-126).

Silva, F. S. D., Caetano, L. A. O., Silveira, C. A. B., \& Junqueira, C. R. A. (2019). A intervenção grupal e o uso da arte como ferramentas produtivas para pessoas com Alzheimer. Vínculo, 16(2), 88-109.

Wang, D., Hu, B., Hu, C., Zhu, F., Liu, X., Zhang, J., ... \& Peng, Z. (2020). Clinical characteristics of 138 hospitalized patients with 2019 novel coronavirusinfected pneumonia in Wuhan, China. Jama, 323(11), 1061-1069.

Wolters, F. J., Chibnik, L. B., Waziry, R., Anderson, R., Berr, C., Beiser, A., ... \& Hofman, A. (2020). Twenty-seven-year time trends in dementia incidence in Europe and the United States: The Alzheimer Cohorts Consortium. Neurology, 95(5), e519-e531.

Xavier, A. P. G., \& Alvarez, Â. M. (2006). Avaliação da assistência prestada via telefone aos familiares de portadores da doença de Alzheimer. Extensio: Revista Eletrônica de Extensão, 3(4).

Ximenes, M. A., Rico, B. L. D., \& Pedreira, R. Q. (2014). Doença de Alzheimer: a dependência e o cuidado. Revista Kairós: Gerontologia, 17(2), 121-140. 